\title{
Development of a teletechnology protocol for in-home rehabilitation
}

\author{
Helen Hoenig, MD; ${ }^{1-2 *}$ Jon A. Sanford, MArch; ${ }^{3}$ Tina Butterfield, OTR; ${ }^{3}$ Patricia C. Griffiths, PhD; ${ }^{3}$ \\ Peg Richardson, OTR/L; ${ }^{1}$ Katina Hargraves, BS ${ }^{1}$ \\ ${ }^{1}$ Durham Department of Veterans Affairs Medical Center (VAMC), Physical Medicine and Rehabilitation Service, \\ Durham, NC; ${ }^{2}$ Center on Aging and Human Development, Duke University, Durham, NC; ${ }^{3}$ Atlanta VAMC, Rehabilita- \\ tion Research and Development Service, Decatur, GA
}

\begin{abstract}
Our ability to provide in-home rehabilitation is limited by distance and available personnel. We may be able to meet some rehabilitation needs with videoconferencing technology. This article describes the feasibility of teletechnology for delivering multifactorial, in-home rehabilitation interventions to community-dwelling adults recently prescribed a mobility aid. We used standard telephone lines to provide twoway video and audio interaction. The interventions included prescription of and/or training in functionally based exercises, home-hazard assessment, assistive technology, environmental modifications, and adaptive strategies. Patients were evaluated in three transfer and three mobility tasks, and appropriate treatment was provided over the course of four visits. To date, 13 of the 14 subjects enrolled in the rehabilitation study have completed all four visits (56 visits total). Equipment-related problems were most common early in the study, particularly on the initial visit to a subject's house. We identified (mean \pm standard deviation [SD]) $13.1 \pm 7.9$ mobility/self-care problems per subject and made $12.5 \pm 8.3$ recommendations per subject to address those problems. At 6-week follow-up, 60.1 percent of our recommendations had been implemented. The greatest number of problems was identified for tub transfers (mean \pm $\mathrm{SD}=3.4 \pm 1.4$ ), the greatest number of recommendations was made for toilet transfers (mean $\pm \mathrm{SD}=3.1 \pm 3.4$ ), and the most frequently implemented recommendations were for transition between locations. Overall, our results show promise that both the telerehabilitation technology and intervention procedures are feasible.
\end{abstract}

Key words: adaptive strategies, assistive technology, homehazard assessment, home-health services, home modification, occupational therapy, physical therapy, telehealth, telerehabilitation, videoconferencing.

\section{INTRODUCTION}

Home healthcare has subsumed an increasingly important role in healthcare over the last 20 years. Short hospital stays often result in patients being discharged to their homes before attaining maximal function [1-2]; yet travel for follow-up outpatient therapy may be too difficult, too expensive, or not possible [1], particularly for people with mobility limitations [3]. To compensate, we use in-home rehabilitation to ensure patients continue to progress and their skills generalize to the home environment. However, in-home rehabilitation offers a number of challenges. First, the therapist providing in-home rehabilitation is seldom the same therapist or even employed by the same institution from which the patient received initial rehabilitation, which undermines the continuity of care. Second, availability of therapists in homecare settings may be limited, particularly in rural areas.

\footnotetext{
Abbreviations: ADL $=$ activities of daily living, $\mathrm{AT}=$ assistive technology, FIM = Functional Independence Measure, ISDN = integrated services digital network, OT = occupational therapist, POTS = plain old telephone system, PS = private sector, $\mathrm{PT}=$ physical therapist, $\mathrm{SD}=$ standard deviation, $\mathrm{V}=$ veteran, VA $=$ Department of Veterans Affairs, $\mathrm{VAMC}=\mathrm{VA}$ medical center.

*Address all correspondence to Helen Hoenig, MD; Physical Medicine \& Rehabilitation Service, Durham VA Medical Center, 508 Fulton Street, Durham, NC 27705; 919-2866874; fax: 919-416-5913. Email: helen.hoenig@med.va.gov DOI: 10.1682/JRRD.2004.07.0089
} 
Finally, costs for home healthcare are substantial and expected to nearly triple by 2014 ( www.cms.hhs.gov) [4].

Videoconferencing technology, which permits realtime two-way video and audio interaction between provider and patient, is potentially a cost-effective method for meeting some of the needs for in-home rehabilitation. Occupational therapist (OT) and physical therapist (PT) interventions that may be suitable for telerehabilitation include assessment of the home environment and training in adaptive strategies. This article describes our use of remote technology to deliver a telerehabilitation intervention to patients at home and the selection and adaptation of intervention strategies that complement the remote technology. We performed a literature review that guided the selection of the technology and interventions and their feasibility for the telerehabilitation arm of a larger, ongoing randomized trial.

\section{LITERATURE REVIEW}

\section{Teletechnology}

Historically, store-and-forward teletechnologies that transmit data, still images, or video recordings [5-7] have been used for providing consultative services and monitoring chronic-health conditions [8-10]. However, rehabilitation depends on real-time two-way observation, communication, and interaction between the therapist and the patient. Satellite telemedicine facilities allow for live two-way communication with high-definition images that are especially useful for management of complex medical conditions but require costly specialized videoconferencing equipment (i.e., high-resolution or integrated services digital network [ISDN] communications technology) [11-15]. Moreover, satellite telemedicine facilities do not provide access to the home environment for evaluation of physical function and prescription of adaptive strategies. Clinicians have successfully adapted videoconferencing technology to in-home rehabilitation using fixed video cameras to provide realtime two-way communication for treating mental illness [16] and monitoring skin ulcers [15,17-19]. However, assessment and treatment of functional impairment is optimally carried out in the context of the functional activity itself (e.g., bathing in the bathroom or cooking in the kitchen). As a result, telerehabilitation necessitates a mobile rather than fixed camera for monitoring activity.
Our literature review found only three studies that used videoconferencing technology for assessing functional activities, gait/mobility, and home safety. One small study reported that a group treated in the home with videoconferencing technology experienced significantly greater improvements in Functional Independence Measure (FIM) scores than individuals who received traditional in-home care [20]. Two additional studies demonstrated that videoconferencing technology could be used successfully for developing home-modification recommendations and that videoconferencing equipment produced images sufficient for visualizing and assessing all but fine motor movements (resolution sufficient for therapists at a remote location to determine space measurements in a home with 1/4 in. accuracy) [21-22].

\section{In-Home Rehabilitation Interventions}

A variety of rehabilitation interventions are used in the home as a part of standard rehabilitation home-health practices, including exercise, assistive technology (AT), adaptive strategies, and environmental modifications.

\section{Exercise}

Three studies examined the effects of home-based exercises. Campbell et al. reported favorable results from a focused exercise intervention prescribed by a PT during a home visit and independently carried out by participants [23]. Similarly, Tinetti et al. found positive effects from exercise prescribed by a PT in the home as a part of a multifactorial intervention [24]. Gill et al. found that an adaptation of Tinetti's program reduced the rate of functional decline among frail community-dwelling elders [25].

\section{Adaptive Strategies and Assistive Technology}

We found no studies that examined use of adaptive methods in the home separate from use of AT. Two studies have reported benefits from providing AT through home visits. Chamberlain et al. found that patients receiving a home visit from an OT after discharge from the hospital were more likely to receive equipment that fit correctly and to bathe independently [26]. Mann et al. showed that recommendations for AT by an OT reduced the rate of functional decline among homebound older persons [27]. 


\section{Home Modifications}

We found no studies that examined environmental modifications alone, but some clinical trials examined inhome environmental modifications in conjunction with AT and adaptive strategies. For example, in their successful trial, Mann et al. recommended environmental modifications as well as AT [27]. Similarly, Cumming et al. reported a reduction in falls with home modifications recommended in conjunction with an OT home visit [28]. A recent meta-analysis of falls research concluded that interventions reduce home hazards [29].

\section{Process of Care}

Several studies showed that real-time observation of a patient's functional performance and their immediate response to therapeutic recommendations is an important aspect of the rehabilitation process. Compared with novice PTs, experienced PTs tend to respond in real time to the needs and responses of the individual patients [30]. A study of OTs showed that clinical reasoning was often tested in action on a moment-to-moment iterative basis [31]. Observation of performance enables an activity to be broken down into its task components (e.g., approaching the tub, turning on the water, getting into the tub, sitting down in the tub) [32] and is perceived as the only reliable way to understand the complex person-environment interactions that characterize activity of daily living (ADL) performance and develop ecologically based individualized interventions [33].

\section{Conclusions}

Our literature review provided reasonable evidence of the effectiveness of home visits for prescribing functionally based exercises, carrying out home-hazard assessments, and training patients in the use of AT and adaptive strategies. It also supported the importance of real-time interaction between the therapist and the patient. Thus, technological needs for in-home rehabilitation include observation while the patient carries out gross motor activities and activities in diverse locations as well as real-time two-way communication between the patient and the therapist. Cost-effective approaches might include teletechnology operated by the patient and/or home-health providers. Home-health nurses might use teletechnology to provide therapists with important information prior to an OT/PT home visit (increasing both efficiency and effectiveness), facilitate consultation with providers who treated the patient in the hospital (improv- ing coordination of care), and enable cost-effective follow-up after an OT/PT home visit. However, teletechnology, whether used by a home-health nurse or by the patient alone, does not provide face-to-face interaction between the rehabilitation provider and the patient and thus is likely to supplement rather than replace traditional rehabilitative care. Our literature review indicated that wireless videoconferencing technology based on a plain old telephone system (POTS) has the potential to meet in-home rehabilitation needs because it is mobile and allows for two-way communication.

\section{METHODS}

\section{Telerehabilitation Technology: Protocol and Proce- dures}

To match rehabilitation needs to the capabilities of the technology, we needed to (1) identify an appropriate patient population, (2) modify POTS-based teletechnology equipment to permit real-time two-way video and audio interaction in diverse locations in the home, (3) develop procedures to enable use of teletechnology for rehabilitation, (4) develop rehabilitation assessment and intervention protocols for use with teletechnology, and (5) train personnel to deliver the telerehabilitation interventions.

Approval for the study was obtained from the institutional review boards of the Durham Department of Veterans Affairs (VA) Medical Center (VAMC), the Shepherd Center, and the Atlanta VAMC. All patients provided informed consent and a release to use their image.

\section{Target Population}

Based on prior studies showing that home-based interventions can reduce falls [24,29], improve safety among users of bathroom safety equipment [26], and reduce the rate of functional decline among frail elders $[25,27]$, we targeted adults who had recently been prescribed a walker or wheelchair and were residing in the community because this group is at high risk for falls and/or functional decline and likely needs bathroom safety equipment. To ensure patients were able to provide informed consent and follow the directions involved with therapy, we excluded patients with cognitive impairment. In addition, the study sample was further limited to patients living within a 1-hour driving distance from the VAMC so research personnel could conduct the home 
visits. Finally, we excluded patients whose life expectancy was less than 6 months based on medical record review.

Ultimately, telerehabilitation interventions will likely be carried out in conjunction with a home-health agency. However, our goal was to determine the feasibility of the technology and procedures; therefore, all aspects of this study were carried out by research personnel.

\section{Equipment}

The videoconferencing system we used in this clinical trial was developed for a previous study on providing remote home assessments [21] and tailored for this project to allow live two-way video and audio patienttherapist interaction. The system consisted of "off-theshelf" technology that used POTS lines, which are located in virtually all homes in the United States. The remote home-video kit (Figure) was transported to the patient's home for each visit. The remote kit weighs only $15 \mathrm{lb}$ and fits in a computer-sized case. The entire kit costs approximately $\$ 3,500$ and can be operated by a nonspecialist with a few hours of training.

Real-time two-way audio and video were transmitted from a mobile camera, headset, and monitor to and from a remote videophone plugged into the POTS line in the home and from the remote videophone to a base unit videophone (Figure) that was viewed by a therapist in the hospital. Both videophones were "plug n' play" and replaced standard phones without additional installation costs. The videophone in the home used wireless radio frequency technology (Polaris Industries GW 2400, Medina, Montana) that enabled real-time images of the patient to be transmitted from a portable palm-sized remote video camera (Sony DCR-IP78T MicroDV, Sony Corp, New York, New York) to a videophone (StarView 500 TravelStar, StarView Corp, Tucson, Arizona) up to $985 \mathrm{ft}$ away. The images were then transmitted through POTS lines to the base unit videophone (StarView 2000 Pro) located at the VAMC. We selected the MicroDV camera because of its extremely small size and low-light

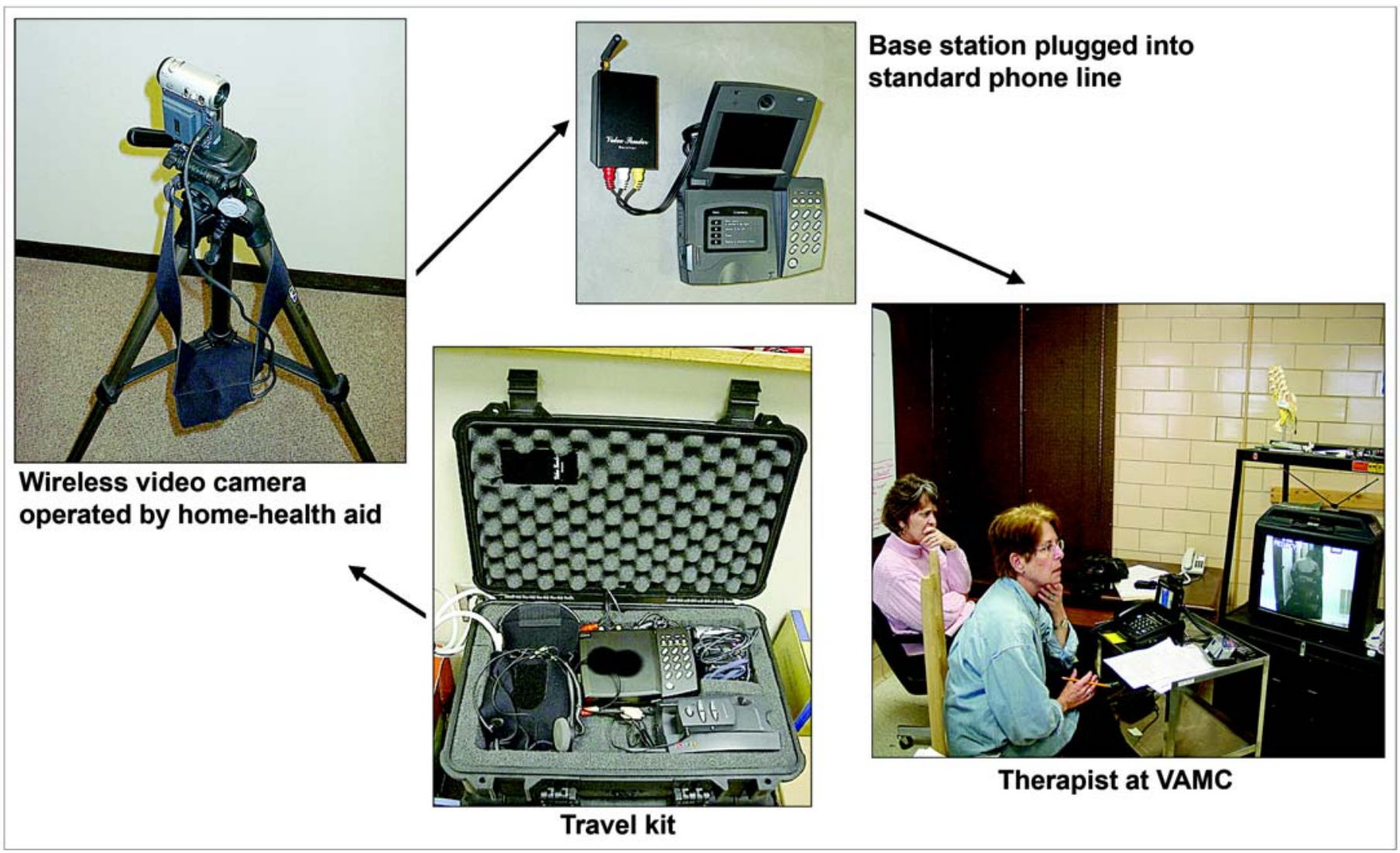

Figure.

Telerehabilitation and communication equipment. VAMC = Department of Veterans Affairs medical center. 
capability. In addition, the camera functions at $0 \mathrm{~lx}$, which minimized the effects of poor household lighting on the quality of the images transmitted to the therapists. We chose the StarView videophone because it is extremely small (6 1/2 in. $\times 43 / 4$ in. $\times 11 / 2$ in.) and the integral monitor folds flat for ease of storage. Finally, a wireless battery-operated 4 in. monitor in combination with a wireless headset enabled the therapist to have twoway video and audio communication with the patient throughout the home (not shown in Figure). The small size of the monitor allowed for easy transport by patients (e.g., in their lap or walker basket) as they engaged in activities throughout the home. The wireless signals from the headset, monitor, and camera could penetrate walls and other obstructions, which enabled the remote videophone to remain in the case in a convenient fixed location while the camera operator moved freely about the home. This permitted the therapist at the hospital to observe the subject throughout the entire home.

\section{Procedures}

At the beginning and end of each session, the therapist interacted directly with the patient. However, during task performance, the technician, who was in continuous communication with the therapist via headset, was the primary person communicating with and directing the patient through the relevant protocols. This allowed the patient to concentrate on performing each task safely without being distracted by the technology. During task performance, the patient was able to see and hear the therapist via the wireless monitor. In addition, the therapist was able to view the patient live via the video camera and hear all communication between the technician and patient.

To augment the real-time communication with the therapist, we also videotaped the patient's performance. This provided opportunities for subsequent consultation as needed (e.g., recommendations for home modifications could be reviewed by an architect). In addition, it provided a back-up record of the patient's performance in the event the live telecommunication failed during the home visit.

\section{Assessment Protocols}

Although POTS-based videoconferencing technology has advanced significantly in the past several years, it still lacks the resolution of more expensive high-speed ISDN equipment. As a result, the equipment was bestsuited for activities that required gross motor skills, such as mobility and transfer tasks. Therefore, we focused on mobility tasks (kitchen mobility, transition between locations, and enter/exit the home) and transfer tasks (toilet, tub/shower, and bed).

The assessment began with the classification of patients into one of four basic functional mobility groups. We classified patients using a derivation of the physical performance measure developed by Guralnik et al., which predicts disability and nursing home placement [34-35]. Based on success or failure with a single chair rise and side-by-side stance, patients were classified into one of four mutually exclusive groups that represented increasing skill levels. The groups included: unable to sit, able to sit but unable to stand, able to stand but unable to walk, able to walk. We used group assignment to match patients to appropriate intervention strategies. Following assignment to one of the four groups, we evaluated patients' performances in each of the three transfer and mobility tasks, which they completed in their usual and customary manner. We selected the tasks based on those measured in the motor FIM. The three transfer tasks were toilet, tub/ shower, and bed; the three mobility tasks were kitchen mobility, transition between locations, and enter/exit the home. Stairs were not tested because they are not present in many patient homes in Georgia and North Carolina.

\section{Intervention Protocols}

The interventions comprised several components that targeted both the individual and the home environment. Specifically, an exercise component targeted underlying organ-system impairment, while adaptive strategies targeted extant disability by either increasing an individual's functional abilities (i.e., adaptive methods and AT) or environmental demands on the individual (i.e., home modifications). We designed the interventions to be similar to PT/OT practices typical of home healthcare for patients with nonspecific functional decline and/or impaired mobility. The entire protocol consisted of four visits. The teletechnology equipment was brought to the house by the technician for each visit. Two of the visits focused on functional mobility, another visit focused on exercise training, and the final visit addressed any remaining issues.

\section{Exercise}

The exercise component used functionally based exercises because both PTs and OTs commonly use such exercises and the literature suggests that they may be 
especially useful for treating disability in the older population $[32,36]$. The exercise intervention was not progressive because of both the limited number of treatment visits and a prior study that found few patients actually progressed to higher levels, despite an average of 16 PT home visits over a 6-month period [25]. An expert panel of clinicians (2 OTs, 1 PT, and a geriatric rehabilitation physician) selected specific exercises based on successful clinical trials and their own clinical experiences [2425,37-38]. Several exercises were identified for each of the four functional mobility levels, with some exercises being applicable to more than one level. Exercises at each level had resistive, power, and balance components. Detailed protocols for study personnel and patient handouts were developed for each exercise. Exercises were prescribed on the second intervention visit; exercise performance and any problems with the exercises were reviewed on the third and fourth visits.

\section{Adaptive Strategies}

The adaptive strategies component of the intervention included three types of strategies: adaptive methods (e.g., one-handed dressing techniques, standing pivot transfer), assistive technology (e.g., walker), and environmental modifications (e.g., removing throw rug). For each of the transfer (toilet, tub/shower, and bed) and mobility tasks (kitchen mobility, transition between locations, and enter/ exit the home), a set of potentially useful interventions was developed for each of the four groups (i.e., from unable to sit to able to walk). The interventions were developed based on a review of relevant publications and the input of our expert panel, along with an architect specializing in AT and home modification [39-43].

Detailed protocols were developed for study personnel that specified barriers to patient's task performance and specific adaptive methods, assistive devices, and environmental modifications that may be considered. For example, the protocol for toileting includes the following procedures: checking toilet and AT (e.g., grab bars, transfer board) to ensure appropriate height and sturdiness and observation of the patient transfer on and off the toilet. If the evaluation shows that additional equipment is needed, it is requested and the patient is subsequently reassessed with the new equipment. The environmental barriers assessed while getting on and off the toilet include: floor surface, lighting, presence of grab bars, and maneuvering space. Adaptive strategies to overcome these toiletingrelated barriers include: adaptive methods such as using a different bathroom or a urinal and mobility and transfer training; AT such as a raised toilet seat, a safety frame, or a commode chair; and home modifications such as additional lighting, grab bars, and replacing the toilet.

All recommendations by the study therapist are noted on an Adaptive Prescription Record for study purposes, and the therapist documents patient progress at each visit in the patient medical record in accordance with hospital policy. The Appendix available online only at https:// www.rehab.research.va.gov) provides a sample Adaptive Prescription Record for the toilet-transfer task. The recommendations for each functional task and mobility skill level are then incorporated into the Adaptive Prescription Record, which is given to the patient. In addition to the protocols for the study personnel and the Adaptive Prescription Form, we developed patient handouts for commonly used adaptive strategies and technologies based on clinical experience and protocols from other relevant studies (available on request) [25,27,43-44].

\section{Personnel}

Our goal was to develop interventions and technologies that either a PT or OT could easily use with the support of a home-health agency's nurse's aid or nurse. Project therapists were all licensed in their relevant discipline, with at least 2 years of clinical experience. Technicians with limited training operated the equipment in the home, which mimics, under research conditions, the type of support that might be available in the home from a Certified Nurse Assistant with a private home-health agency. None of the technicians who operated the equipment in the patient's home had rehabilitation training, although they were experienced research assistants that had completed all required human subjects and Health Insurance Portability and Accountability Act training and were trained in collection of self-report and performance-based measures. In addition, the technicians received training in the study protocols, body mechanics, transfer techniques, and basic cardiopulmonary resuscitation. All personnel (therapists and technicians) met Joint Commission on Accreditation of Healthcare Organizations training requirements for providing care in the home. As noted earlier, the technician hooked up the remote videophone to the POTS in the patient's home, operated the video camera, and provided the immediate direction to the patient during functional activities, while maintaining continuous communication with the therapist. A research engineer was available on-call for equipment-related problems. 


\section{RESULTS}

\section{Feasibility of Wireless Telerehabilitation Technology and Procedures}

The larger randomized trial from which the telerehabilitation sample of patients was derived is enrolling patients in usual care, in-home rehabilitation, and telerehabilitation arms at a ratio of 2:1:1, because the primary research question in the larger study is the effect of usual care versus either in-home rehabilitation or telerehabilitation, with the secondary (exploratory) research question being usual care versus telerehabilitation. This study reports solely on the feasibility of the technology and interventions developed for the telerehabilitation arm. To date, $54 \mathrm{~V}$ and 7 PS patients have been enrolled in the randomized trial, of whom 16 were in the telerehabilitation group; 13 of the 16 patients enrolled in the telerehabilitation group have completed all intervention visits and 6-week follow-up (one dropped out after 2 visits and two others are in progress), and these 13 patients are the focus of this report.

The mean age of the telerehabilitation patients was 58.2 years (range 42-79 yr) (Table 1). The majority of telerehabilitation patients (69.2\%) were married. At baseline, the proportion of patients who reported they needed help with ADL ranged from 0 percent for using the refrigerator to 15.4 percent for toilet transfers to 38.5 percent for tub transfers and enter/exit the home. The proportion of patients who reported difficulty with self-care tasks ranged from 23.1 percent for using the refrigerator to 61.5 percent for toilet transfers to 76.9 percent for tub transfers. The patients reported using a wide variety of mobility aids (69.2\% cane, $61.5 \%$ walker, $38.5 \%$ wheelchair, $15.4 \%$ crutches), and 75.0 percent reported using more than one mobility aid.

Table 1.

Patient demographic characteristics $(N=13)$.

\begin{tabular}{lc}
\hline \multicolumn{1}{c}{ Characteristic } & $\begin{array}{c}\text { Percentage or } \\
\text { Mean } \pm \text { SD }\end{array}$ \\
\hline White & 53.8 \\
Education: High School Graduate & 92.3 \\
Income: Less than \$15,000/yr & 38.5 \\
Married & 69.2 \\
Live Alone & 15.4 \\
Male & 76.9 \\
Age & $58.2 \pm 12.1$ \\
${ }^{*}$ Range 42-79 yr. & \\
SD = standard deviation. & \\
\hline \hline
\end{tabular}

Overall, the equipment and protocols functioned well. Equipment-related problems were most common early in the study and on the initial visit to the subject's home. Of the 13 telerehabilitation patients who completed all four visits (56 visits total), equipment malfunctions occurred with three patients on a total of four occasions. Of these, two of the problems occurred on visit one with two different patients and two of the problems occurred with the same subject on visits three and four. Reasons for the malfunctions were (1) electrical storms interfering with wireless transmission in the home $(n=2)$ and (2) the remote station in the home and the base station at the hospital were set to different channels $(n=2)$. On another occasion, the camera battery was incompletely charged and the video, but not the audio, was lost at the end of the session. On one other occasion, the call-waiting system on the patient's phone interrupted a treatment session and a second call had to be placed to complete the session. These problems were corrected by waiting for adverse weather to clear, checking that all equipment was operational and fully charged prior to departure, and temporarily disabling any call-waiting or call-blocking telephone systems prior to starting the call.

Table 2 shows the problems, recommendations, and proportion of recommendations implemented by type of task. On average, we identified (mean \pm standard deviation [SD]) $13.1 \pm 7.9$ problems per patient and made $12.5 \pm 8.3$ recommendations per patient to address those problems. At 6-week follow-up, 60.1 percent of the recommendations had been implemented. The greatest number of problems was identified for tub transfers (mean $\pm \mathrm{SD}=$ $3.4 \pm 1.4$ ), the greatest number of recommendations was made for toilet transfers (mean $\pm \mathrm{SD}=3.1 \pm 3.4$ ), and the recommendations most frequently implemented were for transition between locations. Table $\mathbf{3}$ shows the types of recommendations made by task. The mean number of recommendations per patient, by intervention, ranged from 5.54 for environmental modifications to 4.69 for AT to 2.62 for adaptive strategies. The proportion of recommendations implemented for specific types of recommendations ranged from 46 percent for environmental modifications to 53 percent for AT to 89 percent for adaptive strategies. The patients reported participating in an exercise program an average of $5.2 \pm$ SD $2.5 \mathrm{~d} / \mathrm{wk}$.

We obtained qualitative data using a questionnaire with several open-ended questions completed during the 6-week follow-up visit. All of the patients (100\%) reported telerehabilitation was "easier" than traveling to 
JRRD, Volume 43, Number 2, 2006

Table 2.

Problems, recommendations, and recommendations implemented by task.

\begin{tabular}{lccc}
\hline \multicolumn{1}{c}{ Task } & $\begin{array}{c}\text { Problems } \\
\text { (Mean } \pm \text { SD) }\end{array}$ & $\begin{array}{c}\text { Recommendations } \\
\text { (Mean } \pm \text { SD) }\end{array}$ & $\begin{array}{c}\text { Recommendations } \\
\text { Implemented } \\
\text { (Percentage) }\end{array}$ \\
\hline Toilet Transfer & $2.5 \pm 1.3$ & $3.1 \pm 3.4$ & 44.5 \\
Tub Transfer & $3.4 \pm 1.4$ & $2.8 \pm 1.6$ & 66.1 \\
Bed Transfer & $1.6 \pm 2.0$ & $1.3 \pm 1.4$ & 41.0 \\
Transition Between Locations & $1.6 \pm 1.7$ & $1.4 \pm 1.6$ & 77.8 \\
Enter/Exit Home & $3.1 \pm 2.3$ & $1.8 \pm 1.5$ & 60.0 \\
Using Refrigerator & $0.9 \pm 1.0$ & $2.1 \pm 2.1$ & 48.2 \\
All Tasks & $13.1 \pm 7.9$ & $12.5 \pm 8.3$ & 60.1 \\
\hline SD $=$ & &
\end{tabular}

SD = standard deviation.

Table 3.

Number of recommendations and percentage implemented by intervention type.

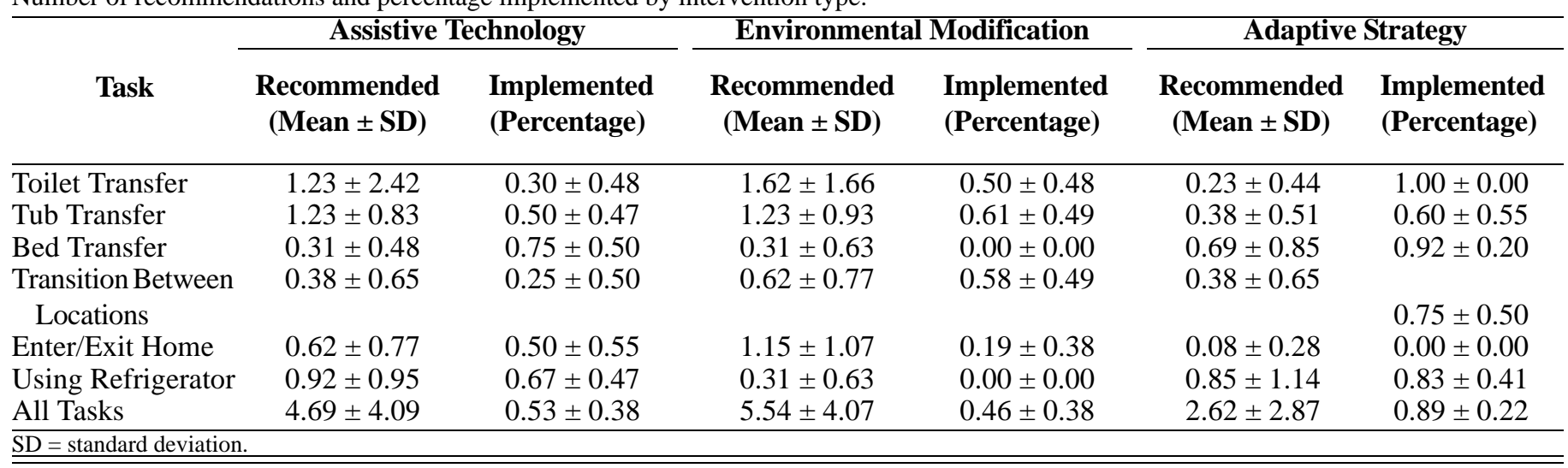

the VAMC for therapy. Likewise, they all reported positive benefits from the therapy. Patients reported liking the convenience $(72.7 \%)$, interactivity $(54.5 \%)$, progressive nature of the experience (63.6\%), and increased one-onone attention (27.3\%). In response to the question "In what ways did the video therapy help you?” 63.6 percent of patients mentioned learning new techniques, learning the right versus wrong way to do tasks, or gaining a new awareness of how to perform the activities. Twenty-five percent of patients reported enjoying the staff (either mentioning particular members or interaction with the telerehabilitation staff as a whole). In response to an open-ended question about dislikes, 90.1 percent of the sample had no dislikes and 9.1 percent disliked having to schedule time for the video crew to come over.

We obtained qualitative data from study therapists as well using a similar questionnaire administered after the final telerehabilitation visit. The telerehabilitation protocols were implemented by four therapists at two sites, and exit interviews were obtained from them on all 13 telerehabilitation patients. The study therapists identified the following benefits from telerehabilitation: ability to interactively work with the patient (45.5\%); ability to see the home environment (63.6\%); ability to observe, assess, and correct unsafe practices (90.0\%); and ability to observe functional mobility in context (54.5\%). The study therapists reported that they liked seeing the assistive devices patients used in the home environment (54.5\%), viewing the space restrictions in certain rooms to inform recommendations (45.5\%), and making the home environment safer (36.4\%). The therapists did not note any problems with telerehabilitation beyond the infrequent equipment-related problems described earlier.

The technology has also been used to enhance continuity of care and facilitate consultation. Therapists and consultants were able to directly view and hear the interaction of the therapist with the patient during the previous treatment sessions. The equipment also facilitated 
coverage during therapists' scheduled and unscheduled leave for 28.6 percent of the telerehabilitation patients and 10.7 percent of the telerehabilitation visits. For one patient, a research therapist located in Atlanta, Georgia, performed two of the scheduled telerehabilitation visits during a period of unexpected sick leave. The therapists used the videotapes from the live telerehabilitation visits to consult with another expert provider (e.g., therapist of another discipline or physician) for 4 of the 13 patients.

\section{DISCUSSION}

Our initial findings suggest that wireless videoconferencing is a feasible way to provide individualized therapy in the home. A feasibility study may be considered a success if positive results are achieved for at least some of the participants. Clearly, positive results were achieved for at least some of the patients, judging by qualitative data from the patients and therapists and by the quantitative data showing the ability of therapists to identify problems and make recommendations, and most importantly, the frequency with which the patients implemented the recommendations.

A key question, which was not directly examined in this is feasibility study, is how telerehabilitation may compare with in-home visits. However, that question may be addressed indirectly by comparison with other studies. The number and kinds of interventions for the telerehabilitation patients were similar to those provided in-person for mobility and transfer tasks and home hazards in the clinical trial by Mann et al. [27] (mean devices prescribed per subject was 3.9 in the Mann study vs 4.7 in our study). The telerehabilitation patients had implemented the majority of the recommended interventions at 6-week follow-up. Knowing how this compares with inperson in-home care is difficult because some environmental interventions may not have been able to be implemented within 6 weeks (e.g., a ramp) and data on "implementation of recommendations" may not be comparable with "disuse/nonuse of prescribed devices" measured in other studies. One of the most comparable studies is that of Cushman and Scherer, which examined rates of nonuse for various prescribed assistive devices among a mixed population of patients discharged from an inpatient rehabilitation unit [45]. In that study, nonuse of prescribed devices 3 months after discharge ranged from 36 percent for walkers and wheelchairs to 55 percent for grooming aids. Those rates are quite comparable with the implementation rates for our telerehabilitation patients. Multiple therapists in multiple locations successfully used the teletechnology to facilitate continuity of care from one therapist to another and to obtain consultative input. Moreover, qualitative data from both the patients and the providers was positive.

This study has a number of limitations. While the study findings show feasibility for the technology and procedures, they do not establish the clinical benefit. Protocols were developed for patients at all levels of functional mobility. While the feasibility should not differ for patients with greater impairment, the 13 patients in this study were all ambulatory with an assistive device. Finally, feasibility was established by trained research personnel in only two locations. Results may differ in other locations or with personnel with different kinds of training.

The interventions developed in this study for wireless videoconferencing have a number of potential applications. Reports by home-health therapists and empirical data indicate that it is not uncommon for patients to be sent home with equipment that does not fit and/or is incorrectly used [46-48]. A home-health therapist can provide in-person onsite assessment, which cannot be fully duplicated by teletechnology, no matter how sophisticated. But a PT or OT home visit is expensive and locating appropriate personnel can be difficult, particularly in rural areas. Moreover, sending a home-health therapist out to the patient's home does not provide continuity of care with the personnel who saw the patient in the hospital. Wireless videoconferencing technology would allow the therapist who treated the patient in the hospital to work with nursing personnel at a home-health agency and ensure plans are implemented appropriately. Use of teletechnology by a therapist located at a base hospital or a home healthagency, with a nurse or home-health aid in the field, would target expensive onsite therapy visits to the patients who need them the most. In addition, the videoconferencing could help home-health therapists prepare ahead of time, so that they could direct their attention to the most critical areas when they are in the home. Last, but not least, the video recording enables consultation with experts that may not be readily available to a home-health agency (e.g., rehabilitation architect, rehabilitation engineer, or certified AT provider). Thus, the teletechnology and protocols developed for this study will not replace the inpatient, outpatient, or home-health therapist. Rather, they are tools that can facilitate continuity of care with the 
inpatient and outpatient therapists, more efficient care by the home-health therapist, and consultations with experts.

\section{ACKNOWLEDGMENTS}

This material is the result of work supported with resources and the use of facilities at the VA Rehabilitation Research and Development Service, Washington, D.C.

The authors have declared that no competing interests exist.

\section{REFERENCES}

1. Garber SL, Rintala DH, Clearman RR, Hart KA, Fuhrer MJ. Prevalence of pressure sores in a community-based sample of men and women with spinal cord injury. J Am Paraplegia Soc. 1991;14(2):71-82.

2. Pope AM, Tarlov AR. Disability in America: Toward a national agenda for prevention. Washington (DC): National Academy Press; 1991. p. 24-25, 280-281.

3. Hoenig H, Landerman LR, Shipp KM, George L. Activity restriction among wheelchair users. J Am Geriatr Soc. 2003;51(9):1244-51. [PMID: 12919236]

4. Heffler S, Smith S, Keehan S, Borger C, Clemens MK, Truffer C. Trends: U.S. health spending projections for 2004-2014. Bethesda (MD): Health Tracking; 2005. p. 74-85.

5. Loane MA, Bloomer SE, Corbett R, Eedy DJ, Hicks N, Lotery HE, Mathews C, Paisley J, Steele K, Wootton R. A randomized controlled trial to assess the clinical effectiveness of both realtime and store-and-forward teledermatology compared with conventional care. J Telemed Telecare. 2000;6 Suppl 1:S1-3. [PMID: 10793956]

6. Pal B, Laing H, Estrach C. A cyberclinic in rheumatology. J R Coll Physicians Lond. 1999;33(2):161-62. [PMID: 10340266]

7. Zelickson BD, Homan L. Teledermatology in the nursing home. Arch Dermatol. 1997;133(2):171-74. [PMID: 9041829]

8. Dawson A, Cohen D, Candelier C, Jones G, Sanders J, Thompson A, Arnall C, Coles E. Domiciliary midwife support in high-risk pregnancy incorporating telephonic heart rate monitoring: A health technology randomized assessment. J Telemed Telecare. 1999;5(4):220-30. [PMID: 10829372]

9. Perednia DA, Wallace J, Morrisey M, Bartlett M, Marchionda L, Gibson A, Campbell E. The effect of a teledermatology program on rural referral patterns to dermatologists and the management of skin disease. Medinfo. 1998;9 Pt 1: 290-93. [PMID: 10384464]
10. Mehra MR, Uber PA, Chomsky DB, Oren R. Emergence of electronic home monitoring in chronic heart failure: Rationale, feasibility, and early results with the HomMed SentryObserver system. Congest Heart Fail. 2000;6(3):137-39. [PMID: 12029180]

11. Edwards MA, Patel AC. Telemedicine in the state of Maine: A model for growth driven by rural needs. Telemed J E Health. 2003;9(1):25-39. [PMID: 12699605]

12. Lindberg CC. Implementation of in-home telemedicine in rural Kansas: Answering an elderly patient's needs. J Am Med Inform Assoc. 1197;4(1):14-17. [PMID: 8988469]

13. Robinson SS, Seale DE, Tiernan KM, Berg B. Use of telemedicine to follow special needs children. Telemed J E Health. 2003;9(1):57-61. [PMID: 12699608]

14. Ryan P, Kobb R, Hilsen P. Making the right connection: Matching patients to technology. Telemed J E Health. 2003;9(1):81-88. [PMID: 12699611]

15. Kim HM, Lowery JC, Hamil, JB, Wilkins EG. Accuracy of a web-based system for monitoring chronic wounds. Telemed J E Health. 2003;9(2):129-40. [PMID: 12855036]

16. Mielonen ML, Ohinmaa A, Moring J, Isohanni M. Psychiatric inpatient care planning via telemedicine. J Telemed Telecare. 2000;6(3):152-57. [PMID: 10912333]

17. Hauber RP, Jones ML, Temkin AJ, Vesmarovich S, Phillips VL. Extending the continuum of care after spinal cord injury through telerehabilitation. Top Spinal Cord Inj Rehabil. 1999;5(3):11-20.

18. Shultz EK, Bauman A, Hayward M, Holzman R. Improved care of patients with diabetes through telecommunications. Ann N Y Acad Sci. 1992;670:141-45. [PMID: 1309083]

19. Marrero DG, Vandagriff JL, Kronz K, Fineberg NS, Golden MP, Gray D, Orr DP, Wright JC, Johnson NB. Using telecommunication technology to manage children with diabetes: The Computer-Linked Outpatient Clinic (CLOC) Study. Diabetes Educ. 1995;21(4):313-19. [PMID: 7621734]

20. Nakamura K, Takano T, Akao C. The effectiveness of videophones in home healthcare for the elderly. Med Care. 1999;37(2):117-25. [PMID: 10024116]

21. Sanford JA, Jones ML, Daviou P, Grogg K, Butterfield T. Using telerehabilition to identify home modification needs. Assist Technol. 2004;16(1):43-53. [PMID: 15357147]

22. Dreyer NC, Dreyer KA, Shaw DK, Wittman PP. Efficacy of telemedicine in occupational therapy: A pilot study. J Allied Health. 2001;30(1):39-42. [PMID: 11265272]

23. Campbell AJ, Robertson MC, Gardner MM, Norton RN, Tilyard MW, Buchner DM. Randomised controlled trial of a general practice programme of home based exercise to prevent falls in elderly women. BMJ. 1997;315(7115): 1065-69. [PMID: 9366737]

24. Tinetti ME, Baker DI, McAvay G, Claus EB, Garrett P, Gottschalk M, Koch ML, Trainor K, Horwitz RI. A multifactorial intervention to reduce the risk of falling among 
elderly people living in the community. N Engl J Med. 1994;331(13):821-27. [PMID: 8078528]

25. Gill TM, Baker DI, Gottschalk M, Peduzzi PN, Allore H, Byers A. A program to prevent functional decline in physically frail, elderly persons who live at home. N Engl J Med. 2002;347(14):1068-74. [PMID: 12362007]

26. Chamberlain MA, Thornley G, Stowe J, Wright V. Evaluation of aids and equipment for the bath: II. A possible solution to the problem. Rheumatol Rehabil. 1981;20(1):38-43. [PMID: 7221401]

27. Mann WC, Ottenbacher KJ, Fraas L, Tomita M, Granger $\mathrm{CV}$. Effectiveness of assistive technology and environmental interventions in maintaining independence and reducing home care costs for the frail elderly. A randomized controlled trial. Arch Fam Med. 1999;8(3):210-17.

[PMID: 10333815]

28. Cumming R, Thomas M, Szonyi G, Salkeld G, O’Neill E, Westbury C, Frampton G. Home visits by an occupational therapist for assessment and modification of environmental hazards: A randomized trial of falls prevention. J Am Geriatr Soc. 1999;47(12):1397-1402. [PMID: 10591231]

29. Gillespie LD, Gillespie WJ, Robertson MC, Lamb SE, Cumming RG, Rowe BH. Interventions for preventing falls in elderly people. Cochrane Database Syst Rev. 2003;(4): CD000340. [PMID: 14583918]

30. Jensen GM, Shepard KF, Gwyer J, Hack LM. Attribute dimensions that distinguish master and novice physical therapy clinicians in orthopedic settings. Phys Ther. 1992;72(10):711-22. [PMID: 1528964$]$

31. Fleming $\mathrm{MH}$. Clinical reasoning in medicine compared with clinical reasoning in occupational therapy. Am J Occup Ther. 1991;45(11):988-96. [PMID: 1793122]

32. Morris JN, Fiatarone M, Kiely DK, Belleville-Taylor P, Murphy K, Littlehale S, Ooi WL, O’Neill E, Doyle N. Nursing rehabilitation and exercise strategies in the nursing home. J Gerontol A Biol Sci Med Sci. 1999;54(10):M494-500. [PMID: 10568531$]$

33. Rogers JC, Holm MB, Stone RG. Evaluation of daily living tasks: The home care advantage. Am J Occup Ther. 1997;51(6):410-22. [PMID: 9164607]

34. Guralnik JM, Simonsick EM, Ferrucci L, Glynn RJ, Berkman LF, Blazer DG, Scherr PA, Wallace RB. A short physical performance battery assessing lower extremity function: Association with self-reported disability and prediction of mortality and nursing home admission. J Gerontol. 1994;49(2):M85-94. [PMID: 8126356]

35. Guralnik JM, Ferrucci L, Simonsick EM, Salive ME, Wallace RB. Lower-extremity function in persons over the age of 70 years as a predictor of subsequent disability. N Engl J Med. 1995;332(9):556-61. [PMID: 7838189]

36. Bean JF, Kiely DK, Herman S, Leveille SG, Mizer K, Frontera WR, Fielding RA. The relationship between leg power and physical performance in mobility-limited older people. J Am Geriatr Soc. 2002;50(3):461-67.

[PMID: 11943041]

37. Alexander NB, Galecki AT, Grenier ML, Nyquist LV, Hofmeyer MR, Grunawalt JL, Madell JL, Fry-Welch D. Task-specific resistance training to improve the ability of activities of daily living-impaired older adults to rise from a bed and from a chair. J Am Geriatr Soc. 2001;49(11): 1418-27.

38. Schnelle JF, MacRae PG, Ouslander JG, Simmons SF, Nitta M. Functional Incidental Training, mobility performance, and incontinence care with nursing home residents. J Am Geriatr Soc. 1995;43(12):1356-62. [PMID: 7490386]

39. Cook AM, Hussey SH. Assistive Technologies: Principles and practice. 2nd ed. Philadelphia (PA): Mosby; 2002. p. 1-523.

40. Olson DA, Deruyter F. Clinician's guide to assistive technology. Philadelphia (PA): Mosby; 2002. p. 1-484.

41. Ellwood PM. Transfers-Method, equipment, and preparation. In: Kottke FJ, Lehmann JF, editors. Krusen's handbook of physical medicine and rehabilitation. Philadelphia (PA): Saunders; 1990. p. 529-47.

42. Leslie LR. Training for functional independence and training in homemaker activities. In: Kottke FJ, Lehmann JF, editors. Krusen's handbook of physical medicine and rehabilitation. Philadelphia (PA): Saunders; 1990. p. 56479.

43. Koch M, Gottschalk M, Baker DI, Palumbo S, Tinetti ME. An impairment and disability assessment and treatment protocol for community-living elderly persons. Phys Ther. 1994;74(4):286-94. [PMID: 8140142]

44. Rogers JC. The occupational therapy home assessment: The home as a therapeutic environment. J Home Health Care Pract. 1989:2:73-81.

45. Cushman LA, Scherer MJ. Measuring the relationship of assistive technology use, functional status over time, and consumer-therapist perceptions of ATs. Assist Technol. 1996;8(2):103-9. [PMID: 10163929]

46. Edwards NI, Jones DA. Ownership and use of assistive devices amongst older people in the community. Age Ageing. 1998;27(4):463-68. [PMID: 9884003]

47. Gitlin LN, Levine R, Geiger C. Adaptive device use by older adults with mixed disabilities. Arch Phys Med Rehabil. 1993;74(2):149-52. [PMID: 8431098]

48. George J, Binns VE, Clayden AD, Mulley GP. Aids and adaptations for the elderly at home: Underprovided, underused, and under maintained. Br Med J (Clin Res Ed). 1988;296(6633):1365-66. [PMID: 3134988$]$

Submitted for publication July 29, 2004. Accepted in revised form October 11, 2005. 